\title{
Visual complexity and memorability of maps
}

\author{
Arzu Çöltekin ${ }^{\mathrm{a}, \mathrm{b} *}$, Dario Oertle ${ }^{\mathrm{b}}$, Alžběta Brychtováb \\ a Institute of Interactive Technologies, University of Applied Sciences and Arts Northwestern Switzerland, arzu.coltekin@fhnw.ch \\ ${ }^{b}$ Department of Geography, University of Zurich, Switzerland \{dario.oertle, alzbeta.brychtova\} @gmail.com \\ * Corresponding author
}

Keywords: Visual complexity, computational measures, human perception

\begin{abstract}
:
There are many efforts to quantify visual characteristics of an image via measures of visual saliency (Itti \& Koch, 2001), visual clutter, entropy (Rosenholtz \& Nakano, 2007), and more recently, memorability (Bainbridge, 2020). Taken together, these measures can be studied under the umbrella of visual complexity. Because there is considerable evidence that attention and memory are tightly coupled (Chun \& Turk-Browne, 2007), to characterize visual complexity, we need to examine both measures of attention and measures of recall / memorability. Efforts to quantify visual complexity are interesting both from fundamental and applied science perspectives as they help us understand human visual behavior better, and can help utilize or design visual 'products' in more ergonomic and effective ways.
\end{abstract}

Even though abovementioned vision-based computational approaches are developed with natural scenes in mind and assume 'free viewing' (i.e., looking at the scene of interest without a specific task), both bottom-up (stimulus-driven) and top-down (cognition-driven) models of attention have been previously proposed (Borji \& Itti, 2012; Torralba, Oliva, Castelhano, \& Henderson, 2006). Visuospatial information displays, including maps and map-like displays, are different from the images of natural scenes in that they are abstracted to different degrees and intentionally designed, e.g., they contain elements that might suppress or highlight information to adapt to a specific purpose (such as navigation, environmental analyses, journalism etc.), and/or the characteristics of the users (e.g., children, older adults, business professionals etc.). Information displays are also different from natural scenes in that they rarely are 'meant' for free viewing. Thus, algorithmic approaches that are tested on natural scenes may or may not directly work with visuospatial information displays such as maps (Brychtová, Çöltekin, \& Paszto, 2016; Brychtová \& Çöltekin, 2017). Maps of the physical environment (as opposed to statistical/thematic maps), e.g., navigational or topographic maps, lend themselves well to examine if algorithms from the vision science reliably measure visual complexity of also human-made visuospatial displays, because through satellite imagery we have photographs of the natural scenes as a baseline, as well as the visuospatial displays, i.e., maps, designed by cartographers.

Among all information displays, maps are also uniquely interesting from the perspective of how they require the use of visual and spatial memory systems. For example, during navigation, as soon as we decode the information presented on the map, we must make quick comparisons and decisions, or need to remember the routes we learned. Facilitated by such short or long-term recall, map use is a big part of how we acquire and retain spatial knowledge in modern times. In our previous studies we explored measures of map complexity in relation to direct human ranking of complexity at different scales (Schnur, Bektaş, \& Çöltekin, 2017), human experience with maps where high levels of information complexity cannot be avoided (Çöltekin et al., 2016; Krejtz, Çöltekin, Duchowski, \& Niedzielska, 2017), and if clutter and entropy measures correlate with cartographic generalization (Brychtová, Çöltekin, \& Paszto, 2016; Brychtová \& Çöltekin, 2017). Given the above, in this work-in-progress paper, we explore how well the computational approaches to visual complexity (e.g., saliency, clutter, memorability) work with maps vs. satellite images by comparing the outcomes across datasets, methods and contexts. Specifically, we examine 'agreement' between methods for maps and satellite images of 50 urban and 50 rural areas (because urban areas contain more objects and object type variation, thus richer in information), as well as compare these outcomes to empirical findings in an experiment $(n=40)$ in which we collected eye movements and measured participant performance in a set of map-based recall tasks.

\section{References}

Bainbridge, W. A. (2020). The resiliency of image memorability: A predictor of memory separate from attention and priming. Neuropsychologia, 141, 107408. https://doi.org/10.1016/j.neuropsychologia.2020.107408

Borji, A., \& Itti, L. (2012). State-of-the-art in Visual Attention Modeling. IEEE Transactions on Pattern Analysis and Machine Intelligence, 35(1), 185-207. https://doi.org/10.1109/TPAMI.2012.89

\footnotetext{
${ }^{1}$ Affiliation at the time of data collection and main research phase.
} 
Brychtová, A., Çöltekin, A., \& Paszto, V. (2016). Do the visual complexity algorithms match the generalization process in geographical displays? ISPRS - International Archives of the Photogrammetry, Remote Sensing and Spatial Information Sciences.

Brychtová, Alžběta, \& Çöltekin, A. (2017). Towards assessing generalization quality with visual complexity measures. In 28th International Cartographic Conference (ICC2017). Washington DC. https://doi.org/https://doi.org/10.5167/uzh-148794

Chun, M. M., \& Turk-Browne, N. B. (2007). Interactions between attention and memory. Current Opinion in Neurobiology, 17(2), 177-184. https://doi.org/10.1016/j.conb.2007.03.005

Çöltekin, A., Brychtová, A., Griffin, A. L., Robinson, A. C., Imhof, M., \& Pettit, C. (2016). Perceptual complexity of soil-landscape maps: a user evaluation of color organization in legend designs using eye tracking. International Journal of Digital Earth, 1-22. https://doi.org/10.1080/17538947.2016.1234007

Itti, L., \& Koch, C. (2001). Computational modelling of visual attention. Nature Reviews. Neuroscience, 2(3), $194-203$. https://doi.org/10.1038/35058500

Krejtz, K., Çöltekin, A., Duchowski, A., \& Niedzielska, A. (2017). Using coefficient K to distinguish ambient/focal visual attention during map viewing. Journal of Eye Movement Research, 10(2), 1-13. https://doi.org/10.16910/jemr.10.2.3

Rosenholtz, R., \& Nakano, L. (2007). Measuring visual clutter. Journal of Vision, 7, 1-22.

Schnur, S., Bektas, K., \& Çöltekin, A. (2017). Measured and perceived visual complexity: a comparative study among three online map providers. Cartography and Geographic Information Science, 1-17. https://doi.org/10.1080/15230406.2017.1323676

Torralba, A., Oliva, A., Castelhano, M. S., \& Henderson, J. M. (2006). Contextual guidance of eye movements and attention in real-world scenes: the role of global features in object search. Psychological Review, 113(4), 766-786. https://doi.org/10.1037/0033-295X.113.4.766 